
PWE-013 SELECTIVE CYTOTOXICITY OF ABERRANTLY
GLYCOSYLATED MUC1 BINDING-PS1 PHOTOIMMUNOCONJUGATES FOR OESOPHAGEAL ADENOCARCINOMA TARGETED PHOTODYNAMIC THERAPY IN PRIMARY AND METASTATIC DISEASE

doi:10.1136/gutjnl-2012-302514d.13

\begin{abstract}
${ }^{1,2} \mathrm{M}$ A Butt, ${ }^{*}, 4 \mathrm{I}$ Stamati, ${ }^{3} \mathrm{H}$ Pye, ${ }^{4} \mathrm{G}$ Yahioglu, ${ }^{1,2} \mathrm{R}$ J Haidry, ${ }^{5} \mathrm{D}$ Oukrif, ${ }^{2} \mathrm{M}$ R Novelli, ${ }^{3,4} \mathrm{M}$ P Deonarain, ${ }^{1,2}$ L B Lovat. ${ }^{1}$ National Medical Laser Centre, University College London, London, UK; ${ }^{2}$ University College Hospital, London, UK; ${ }^{3}$ Recombinant Antibody Therapeutics Laboratory, London, UK; ${ }^{4}$ PhotoBiotics Ltd, Imperial College London, London, UK; ${ }^{5}$ University College London, London, UK
\end{abstract}

Introduction Targeted photodynamic therapy (PDT) has the potential to overcome current limitations of PDT agents by offering specific tumour kill with reduced side effects. We have previously shown expression of AG-MUC1, an epithelial cancer target bound by the antibody HuHMFG1, in patients with Barrett's with dysplasia, oesophageal adenocarcinoma (OA) and on OE19, an OA cell line. We have recently developed and characterised photoimmunoconjugates (PIC) of Hu-HMFG1 with the photosensitiser PS1 to specifically target PDT to OA. This is the first study to test the cytotoxic efficacy of HuHMFG1:PS1 PIC's against cancer cells in vitro.

Methods Hu-HMFG1 was conjugated with PS1 (PhotoBiotics) using recently optimised methods. Binding of the HuHMFG1:PS1 PIC to the cancer cell lines SKOV-3 (ovarian), MCF-7 (breast), OE-19 (oesophageal) and HT-29 (colon) was examined with flow cytometetry and analysed with FlowJo software. The efficacy of HMFG1:PS1 PICs on these lines were compared with equivalent free PS1 in the presence or absence of laser activation. Power was set to a clinically relevant light dose. Cell viability was measured with standard MTS assay and the plates read on an ELISA plate reader at $490 \mathrm{~nm}$.

Results Flow cytometry confirmed binding of HMFG1:PS1 PIC to SKOV-3, OE19 and MCF-7 but not HT-29 cells. Cell viability counts in all plates were initially corrected for untreated plate controls and then plotted on a log scale to produce dose response curves. This confirmed significantly superior cytotoxic efficacy of HuHMFG1: PS1 over PS1 in SKOV-3 $(\mathrm{F}=104.93, \mathrm{p}=0.00051)$ and $\mathrm{OE}-19$ $(\mathrm{F}=11.13, \mathrm{p}=0.0125)$, and a trend towards effect for MCF-7 $(\mathrm{F}=3.13$, $\mathrm{p}=0.116$ ) cells using linear regression analysis and an $\mathrm{F}$ test to compare treatments. HuHMFG1:PS1 did not kill HT-29 effectively over control $(p=0.84)$ or differ significantly from PS1 in its efficacy $(\mathrm{F}=0.155, \mathrm{p}=0.71)$ confirming cytotoxicity to be limited to HuHMFG1 expressing cells.

Conclusion This pilot study is the first to successfully demonstrate binding and potential cytotoxicity of targeting HuHMFG1:PS1 PICs against $\mathrm{OA}$ cells in vitro. Absence of photosensitiser effect in the negative control (HT29 cells) confirmed selective cytotoxicity of HuHMFG1:PS1 to AG-MUC1 expressing cells. We further demonstrated that HuHMFG1:PS1 can effectively kill other AG-MUC1 expressing tumours which have historically been treated with HuHMFG1 related therapies.

Competing interests None declared

\section{PWE-014 POLO-LIKE KINASE 1 EXPRESSION PREDICTS ANEUPLOIDY IN THE BARRETT'S METAPLASIA- DYSPLASIA-CARCINOMA SEQUENCE}

doi:10.1136/gutjnl-2012-302514d.14

1,2M A Butt, ${ }^{* 1,2}$ R J Haidry, ${ }^{3} \mathrm{D}$ Oukrif, ${ }^{4} \mathrm{~J}$ M Dunn, ${ }^{2} \mathrm{~A}$ Gupta, ${ }^{2} \mathrm{M}$ Bhatt, ${ }^{3} \mathrm{~L}$ Hrynkiewicz, ${ }^{3} \mathrm{~A}$ Waheed, ${ }^{3} \mathrm{C}$ Owen, ${ }^{3} \mathrm{~A}$ Machhada, ${ }^{2} \mathrm{M}$ Rodriguez-Justo, ${ }^{1,2} \mathrm{M}$ R Banks, ${ }^{1,2} \mathrm{~L}$ B Lovat,

${ }^{2,3} \mathrm{M}$ R Novelli. ${ }^{1}$ National Medical Laser Centre, University College London, London, UK;
${ }^{2}$ University College Hospital, London, UK; ${ }^{3}$ University College London, London, UK; ${ }^{4}$ University Hospital Lewisham, London, UK

Introduction Finding an accurate and convenient biomarker for cancer progression in Barrett's (BE) is of high clinical importance. DNA ploidy abnormalities (DNA-PA) are a reliable predictor of cancer risk in BE, but measurement is expensive and scarcely available. We have shown polo-like kinase-1 (PLK1) may act as a surrogate marker of DNA-PA in oesophageal adenocarcinoma (OA) resection specimens. This study aimed to examine the potential of PLK1 in predicting DNA-PA in the metaplasia-dysplasia-OA sequence.

Methods 36 paraffin embedded oesophageal tissue specimens were selected from patients with non-dysplastic BE (NDBE, $n=5$ ), low grade dysplasia (LGD, $\mathrm{n}=5$ ), high grade dysplasia ( $\mathrm{HGD}, \mathrm{n}=12$ ), intramucosal cancer (IMC, $\mathrm{n}=5$ ) and invasive OA (IOA, $\mathrm{n}=9$ ). Sections were mounted and immunostained with 2 PLK-1 antibodies PLK1-M and PLK1-L using an automated system (BONDMAX, Leica) for consistency. Results were reported by two independent expert pathologists blinded to patient status with the Allred scoring system, a composite of the percentage and intensity of staining.

Results Aneuploidy was present in LGD (20\%), HGD (75\%), IMC $(20 \%)$ and IOA (56\%) samples. Using linear regression analysis, Pearson coefficient was calculated for the correlation between DNAPA and mean Allred expression scores for each PLK1 antibody. PLK1$M$ had a higher degree of correlation $\left(r^{2}=0.26, p=0.001\right)$ then PLK1-L $\left(r^{2}=0.22, p=0.004\right)$. Inter-observer analysis with linear $\kappa$ scores confirmed good correlation of PLK1-M ( $\kappa=0.72,95 \%$ CI 0.60 to 0.83 ) and PLK1-L ( $\kappa=0.53,95 \%$ CI 0.38 to 0.68 ), but a Bland-Altman plot found pathologist 2 had a trend to score PLK-L more highly. However, intra-observer analysis confirmed both PLK1-L scores correlated with ploidy status $\left(r^{2}=0.14, p=0.023\right.$ and $r^{2}=0.27$, $\mathrm{p}=0.0016$ ). Pathologists took $90.3 \mathrm{~s} /$ slide (mean) to review and score with the Allred method. Using a mean Allred cut off score of 3.5, the sensitivity and specificity for the detection of aneuploidy were $81.3 \%$ and $75 \%$ for PLK1-M, 93.8\% and $45 \%$ for PLK1-L.

Conclusion This pilot study demonstrated a significant correlation between Allred reporting of PLK1 staining and DNA-PA. PLK1-L appears more sensitive and PLK1-M more specific. PLK1 immunostaining is relatively inexpensive, less work intensive and is more available than current methods to predict DNA-PA. Scoring staining with Allred is rapid and reproducible. In future, PLK1 staining may provide the basis of a more durable biomarker panel to predict DNA ploidy.

Competing interests None declared.

\section{PWE-015 HER2 EXPRESSION IN THE BARRETT'S METAPLASIA- DYSPLASIA-CARCINOMA SEOUENCE AND POTENTIAL TARGETING IN VITRO WITH THE SINGLE CHAIN ANTIBODY FRAGMENT C6.5}

doi:10.1136/gutjnl-2012-302514d.15

\begin{abstract}
${ }^{1,2} \mathrm{M}$ A Butt, ${ }^{* 1,2} \mathrm{R}$ J Haidry, ${ }^{3} \mathrm{D}$ Oukrif, ${ }^{4} \mathrm{H}$ Pye, ${ }^{4,5}$ I Stamati, ${ }^{5} \mathrm{G}$ Yahioglu, ${ }^{2} \mathrm{M}$ RodriguezJusto, ${ }^{1,2} \mathrm{M}$ R Banks, ${ }^{4,5} \mathrm{M}$ P Deonarain, ${ }^{2,3} \mathrm{M}$ R Novelli, ${ }^{1,2} \mathrm{~L}$ B Lovat. ${ }^{1}$ National Medical Laser Centre, University College London, London, UK; ${ }^{2}$ University College Hospital, London, UK; ${ }^{3}$ Research Department of Pathology, University College London, London, UK; ${ }^{4}$ Recombinant Antibody Therapeutics Laboratory, London, UK; ${ }^{5}$ PhotoBiotics Ltd, Imperial College London, London, UK
\end{abstract}

Introduction There is increasing attention on the integration of targeted agents for oesophageal adenocarcinoma (OA) therapy. The most notable example of success of oesophagogastric targeted therapy was the addition of a HER2 targeting agent in the Phase III ToGA study. However, there is limited data on the expression of 